\title{
Acoplamento de Expressão Unidimensional de Recarga a Modelos de Elementos Analíticos
}

J.A.N. BATISTA, E. WENDLAND, H.E. SCHULZ, Departamento de Hidráulica e Saneamento, EESC-USP, Av. Trabalhador Sancarlense 400, 13566-590 São Carlos, SP, Brasil.

Resumo. Este trabalho diz respeito à aplicação do Método de Elementos Analíticos, desenvolvido originariamente para domínios infinitos, a um problema de escoamento subterrâneo caracterizado em domínio semi-infinito. A aplicação de Elementos Analíticos a esses domínios exige a utilização de métodos auxiliares (por exemplo o método de imagens) que, em contra partida, eliminam a necessidade da utilização de elementos convencionais para a representação de efeitos regionais (por exemplo a recarga direta do aqüífero) dando assim lugar à utilização de expressões unidimensionais. A modelagem de problemas em meios porosos com essa técnica reproduz com precisão o campo de escoamento em regiões semi-infinitas.

\section{Introdução}

As formações geológicas por onde escoam os fluidos subterrâneos ocorrem geralmente sobre áreas que se estendem muito além das regiões onde são estudados problemas de escoamento. Comumente, essas formações apresentam-se em formas alongadas, ou seja, estreitas em relação à sua direção de maior comprimento. Neste trabalho é caracterizado um problema de escoamento no aqüífero Barreiras, litoral Nordeste do Brasil. O aqǘfero é representado em uma faixa de domínio semi-infinita e é modelado a partir do Método de Elementos Analíticos. A região abordada tem sido adotada como fonte de captação de água em projetos de abastecimento de comunidades distantes, sendo necessário avaliar as interferências desses projetos sobre o comportamento natural do manancial.

O Método de Elementos Analíticos (da literatura internacional, Analytic Element Method, AEM) é um método computacional para a obtenção de soluções em campos vetoriais utilizando-se para tanto a sobreposição de soluções analíticas elementares. O Método de Elementos Analíticos pode ser aplicado tanto em domínios finitos como em domínios infinitos. Os primeiros passos para o desenvolvido do Método foram dados no início da década de setenta com o objetivo de construir um modelo de escoamento subterrâneo para a avaliação dos efeitos da construção de um canal entre os rios Tennessee e Tombigbee sobre o escoamento nos aqǘferos da região. O projeto demandava do modelo a representação do escoamento tanto em escala regional (inicialmente 80 por $130 \mathrm{~km}$ ) como em escala local [12, 13].

\footnotetext{
${ }^{1}$ Bolsista de doutorado do CNPq, Email: nbatista@sc.usp.br.
} 
Atualmente a aplicação mais extensa em área de abrangência territorial é o Modelo Nacional Holandês de Águas Subterrâneas atribuído a [3] e apresentado em (http://www.tauw.nl/NL/producten/waterbeheer/nagrom/nagrom.htm). O método permite obter informações de escala local mesmo em abordagens de grande escala como essa, devido à representação de efeitos por meio de funções analíticas.

A formulação empregada no desenvolvimento das funções utilizadas como elementos no AEM tem origem em técnicas bastante conhecidas em Águas Subterrâneas e na Teoria Linear da Elasticidade. A descrição do método e aplicações das Integrais de Cauchy e de Elementos de Contorno em teoria elástica é feita, por exemplo, por $[9,10]$. Em Águas Subterrâneas, essas técnicas são apresentadas, por exemplo, por [11]. Distribuições de vórtices foram também amplamente empregadas em diversos problemas em ambas as áreas (eg., [2]).

\section{Formulação do Método}

Os elementos analíticos são representados matematicamente por soluções obtidas para potenciais complexos. Essas expressões contêm parâmetros que são determinados de forma a satisfazer as condições de contorno. O potencial de referência, definido pela somatória das constantes de integração presentes em cada expressão utilizada, é um dos parâmetros a serem determinados no modelo. Tais condições de contorno não são satisfeitas de forma exata, mas de forma tão precisa quanto possível para um dado número de graus de liberdade e natureza dos elementos. O escoamento irrotacional em aqüíferos de uma só camada dispõe de uma formulação bastante amadurecida no AEM e a precisão dos resultados depende somente do desempenho das máquinas utilizadas [14]. Passamos a discutir de maneira breve, modelos de elementos analíticos para aqüíferos de uma só camada $[15,5]$.

Define-se o vetor de descarga específica $\left[\mathrm{LT}^{-1}\right]$ em um aqǘfero plano a partir da lei de Darcy (equação (2.2)) expressa por:

$$
\begin{aligned}
& q_{x}=-K \frac{\partial \phi}{\partial x}, \\
& q_{y}=-K \frac{\partial \phi}{\partial y},
\end{aligned}
$$

onde $K$ é a condutividade hidráulica $\left[\mathrm{LT}^{-1}\right]$ e $\phi$ é a carga hidráulica [L]. O vetor de descarga total $(Q)$ representa a vazão $\left[\mathrm{L}^{2} \mathrm{~T}^{-1}\right]$ que atravessa a espessura de escoamento. A aproximação de Dupuit-Forchheimer considera que o vetor de descarga específica é uniforme em toda espessura do escoamento, assim as componentes $Q_{x}$ e $Q_{y}$ podem ser escritas como $Q_{x}=h q_{x}$ e $Q_{y}=h q_{y}$, onde $h$ é a espessura do escoamento saturado no aqǘfero.

Considerando-se apenas casos onde as propriedades do aqüífero são constantes em regiões de áreas finitas, o potencial de descarga $\left[\mathrm{L}^{3} \mathrm{~T}^{-1}\right]$ existe tanto para aqǘferos confinados como livres na forma da equação (2.2):

$$
Q_{x}=-\frac{\partial \Phi}{\partial x}
$$




$$
Q_{y}=-\frac{\partial \Phi}{\partial y}
$$

Faz-se $\Phi=\frac{1}{2} K h^{2}=\frac{1}{2} K(\phi-b)^{2}$ para $\phi \leq H$ e $\Phi=K H(\phi-b)-\frac{1}{2} K H^{2}$ para $\phi \geq H$, onde $b$ é a cota da base do aqǘfero [L], $h=\phi-b$, é a espessura saturada para aqǘferos livres [L] e $H$, a espessura do aqǘfero confinado [L]. Note-se que a constante $K H^{2} / 2$ na expressão do potencial para escoamento confinado $(\phi \geq H)$ é adotada de forma que o potencial seja válido em aqüíferos onde $\phi=H$, ou seja, o escoamento pode ser considerado tanto confinado como livre.

A "mola mestra" da formulação do Método de Elementos Analíticos é a definição do potencial complexo a partir de integrais singulares em linhas a serem utilizadas como elementos $[15,9,10]$. O potencial complexo (equação (2.3)) é definido por uma componente de energia potencial real $(\Phi)$, definida na equação (2.2), e uma complexa $(\Psi)$, definida pela condição de Cauchy-Riemann (equação (2.4)), que expressa as linhas de corrente do escoamento.

$$
\begin{gathered}
\Omega=\Phi+i \Psi, \\
\frac{\partial \Psi}{\partial x}=-\frac{\partial \Phi}{\partial y} \\
\frac{\partial \Psi}{\partial y}=\frac{\partial \Phi}{\partial x} .
\end{gathered}
$$

As linhas de corrente são expressas em termos de descarga específica pela equação (2.5):

$$
\begin{gathered}
Q_{x}=-\frac{\partial \Psi}{\partial y}, \\
Q_{y}=\frac{\partial \Psi}{\partial x} .
\end{gathered}
$$

Estão caracterizados basicamente dois tipos de elementos unifilares (sejam retilínios ou curvilínios) na formulação do AEM: as line-dipole e as line-doublet. O potencial complexo para uma line-doublet apresenta uma descontinuidade na componente real de $\Omega$, ou seja, o potencial ( $\Psi$ ). Uma line-dipole, por sua vez, exibe descontinuidade na componente imaginária de $\Omega$ ou seja, nas linhas de corrente $(\Psi)$. Geometricamente, as line-dipole são constituídas por dipolos alinhados sobre uma mesma linha. As line-doublets são constituídas por dipolos dispostos lado a lado, todos com a mesma orientação (Figura 1).

Note-se que em ambos os casos, apenas uma componente apresenta descontinuidade enquanto a componente correlata permanece contínua. A discussão completa da formulação de elementos e da aproximação do intervalo deixado pela descontinuidade de $\Omega$ por intermédio de polinômios de ordem menor que três são discutidos em [15]. Janković [6] apresenta aproximações polinomiais de ordem superior por intermédio de polinômios de Chebyshev [4] introduzindo técnicas de sobrespecificação ao AEM. 


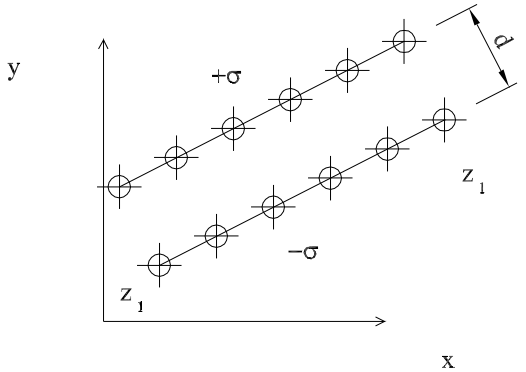

(a)

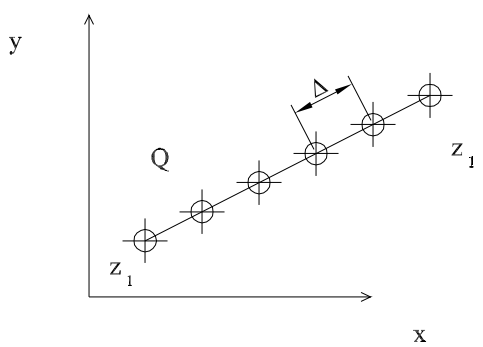

(b)

Figura 1: a) Esquema matemático do elemento line-doublet; b) Esquema matemático do elemento line-sink

O Método pode ser enunciado em termos de um campo vetorial qualquer descrito por intermédio de expressões que possam ser sobrepostas. Um campo vetorial $Q_{i}$ é de maneira geral formulado em termos de divergência e rotacionalidade. Utilizandose a convenção de Einstein para as coordenadas, considere que a rotacionalidade do campo seja representada por $\beta$ e a divergência por $-\gamma$. Em um campo sem divergência, sua descrição é feita apenas pelo gradiente de um escalar chamado função de corrente $(\Psi)$ expresso na equação $(2.6)$ :

$$
\stackrel{\beta}{Q}=-\varepsilon_{i j} \frac{\partial \Psi}{\partial x_{j}},
$$

onde $\varepsilon_{i j}$ é um tensor de segunda ordem. Semelhantemente, o campo irrotacional pode ser representado apenas pelo gradiente do potencial (equação (2.7)):

$$
\underset{i}{Q}=-\frac{\partial \Phi}{\partial x_{i}}
$$

Se os valores de $\beta$ e $\gamma$ são conhecidos, $\Psi$ (equação (2.8)) e $\Phi$ (equação (2.9)) serão então dados pelas seguintes equações:

$$
\begin{aligned}
& \nabla^{2} \Psi=\beta, \\
& \nabla^{2} \Phi=\gamma .
\end{aligned}
$$

De acordo com o teorema de Helmholtz, a porção do campo que não contribui para a rotacionalidade nem para a divergência do campo é escrita em termos do gradiente de um potencial ou em termos do rotacional da função de corrente (equação $(2.10))$ :

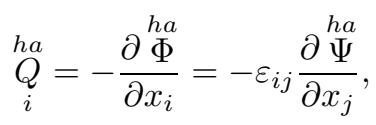

onde $\varepsilon \partial / \partial x_{j}$ representa a rotação e o sobrescrito $h a$ está relacionado ao termo harmônico. Finalmente, a descrição completa de um campo vetorial generalizado (equação (2.11)) é dada pela soma dos três campos: 


$$
Q_{i}=\underset{i}{Q}+\underset{i}{\stackrel{\gamma}{Q}}+\underset{i}{\stackrel{h a}{Q}}
$$

\section{Escoamento Unidimensional da Regarga}

Embora, os aqüíferos possuam geometrias irregulares, a caracterização de aqüíferos estreitos como faixa permite a abordagem de efeitos regionais de forma simplificada eliminando-se a necessidade da utilização de elementos convencionais para a representação da recarga direta do aqüífero.

Além disso, considerando-se um aqüífero onde uma das formações ocorre em maiores proporções, representa-se a formação de maior ocorrência de maneira homogênea (condutividade $K$ e profundidade da base $b$ ) e a recarga $(N)$ uniformemente distribuída. Esquematicamente, a distância entre os contornos e a distribuição de potencial são ilustrados na Figura 2.

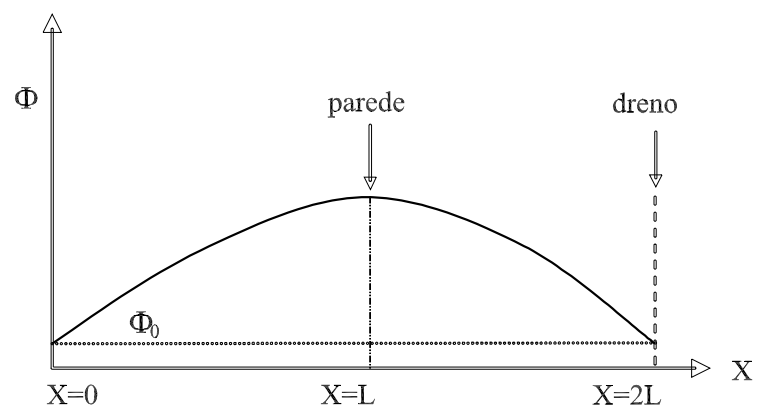

Figura 2: Perfil de potenciais devido à recarga entre dois drenos paralelos

A expressão para esse escoamento é dada pela solução do escoamento unidimensional entre dois drenos (equação (3.1)):

$$
\Phi_{0}=-\frac{N}{2} X^{2}+N L X+\Phi_{0}
$$

onde $L[\mathrm{~L}]$ é a distância entre a fronteira impermeável e a fronteira permeável; $\Phi_{0}$ $\left[\mathrm{L}^{3} \mathrm{~T}^{-1}\right]$ é o potencial de descarga na fronteira permeável $\left(\Phi_{0}=\frac{K}{2} h_{0}^{2}\right)$, onde $h_{0}$ é a carga piezométrica); e $X$ [L] é a posição a partir da fronteira permeável.

A expressão é utilizada no plano horizontal na forma a seguir (equação (3.2)), onde $\xi$ e $\eta$ são as componentes da distância do ponto de referência até a posição considerada no sistema de coordenadas e $\alpha \mathrm{g}$, a rotação das fronteiras em relação à direção $y$.

$$
\Phi_{0}=-\frac{N}{2}(\xi \cos \alpha+\eta \operatorname{sen} \alpha)^{2}+N L(\xi \cos \alpha+\eta \operatorname{sen} \alpha)+\Phi_{0}
$$




\section{O Método de Imagens}

A representação unidimensional da recarga de aqüíferos estreitos abrange uma região semi-infinita enquanto as funções utilizadas no Método de Elementos Analíticos são soluções obtidas para um domínio infinito. A utilização desses elementos em domínios semi-infinitos, por entanto, exige a utilização de métodos auxiliares (e.g. mapeamento conforme, Método de Imagens) para a imposição das condições dos contornos externos ao comportamento do elemento. Neste trabalho o Método de Imagens (MI) é utilizado para a imposição dos contornos de uma faixa infinita que contém a região a ser modelada.

Keller [7] define o Método de Imagens como um método para a construção de funções de Green, para uma região delimitada por planos, em termos de funções de Green válidas em todo espaço (equação (4.1)) e determinou regiões, equações diferenciais e condições de contorno para as quais o método é aplicável.

$$
G\left(r, r^{\prime}\right)=\sum_{r^{\prime \prime} \in S(r)} G *\left(r^{\prime}, r^{\prime \prime}\right) .
$$

A equação descreve a função de Green para dois pontos no espaço, $r$ e sua imagem $r$ '. A função $G^{*}$ por sua vez é definida para "imagens de imagens" $\left(r^{\prime \prime}\right)$ e seus pontos originais.

Keller [7] concluiu que as geometrias das regiões admissíveis ao Método de Imagens para duas e três dimensões são dadas por uma quantidade finita e determinada de planos. Tomando o caso bidimensional como exemplo, apenas os domínios unidos por ângulos da forma $\pi / p$ (onde $p$ é um inteiro) podem ser regiões admissíveis ao Método de Imagens. Dessa forma haverá no máximo quatro retas definindo o domínio.

\section{Acoplamento do Modelo Unidimensional de Re- carga ao AEM}

Sabendo que o Método de Imagens é um método baseado no princípio da sobreposição de soluções, as equações diferenciais para as quais o método é válido devem ser lineares. Sendo o escoamento estacionário de água em meios porosos saturados linear (equações (2.8) e (2.9)), o Método tem sido amplamente empregado no estudo de suas soluções.

Em sua grande maioria o Método de Imagens é aplicado para satisfazer as condições importas ao longo de linhas equipotenciais conhecidas (primeiro tipo) e linhas impermeáveis (segundo tipo). Maxwell (1873) apud Muskat [8], mostra que o Método de Imagens pode também ser aplicado em fronteiras entre regiões homogêneas porém de diferentes condutividades impondo-se simultaneamente condições do primeiro e do segundo tipos. Keller [7] demonstra a aplicabilidade do Método também para condições do terceiro tipo e recentemente, Anderson [1] obteve o mesmo resultado encontrado por Keller [7] utilizando-se o caso particular de escoamento produzido por poços sujeitos a uma fronteira semi-impermeável. 
O acoplamento do Método de Imagens ao Método de Elementos Analíticos é feito estabelecendo-se relações entre os parâmetros dos elementos posicionados na região imaginária e os parâmetros da região original (equações (5.1), (5.2), (5.3), (5.4), (5.5)). Assim, para cada condição de contorno tem-se uma relação.

Para condições do primeiro tipo $(G=0)$ :

$$
\sum \sigma^{\text {imagem }}-\sum \frac{\sigma^{r e a l}}{i}=0
$$

Para condições de contorno do segundo tipo $(\partial G / \partial n=0)$ :

$$
\sum \sigma^{i m a g e m}+\sum \frac{\sigma^{r e a l}}{i}=0
$$

Contornos onde são impostas condições de heterogeneidades (primeiro e segundo tipo) podem ser expressos, para $r^{+}$, por:

$$
\sum \sigma^{\text {imagem }}+\left[\frac{K^{+}-K^{-}}{K^{+}+K^{-}}\right] \sum \frac{\sigma^{\text {real }}}{i}=0
$$

e para $r^{-}$, por:

$$
\sum \sigma^{\text {imagem }}+\left[\frac{2 K^{+}}{K^{+}+K^{-}}\right] \sum \frac{\sigma^{\text {real }}}{i}=0 .
$$

Em contornos onde é imposta a condição do terceiro tipo a relação entre os subdomínios é obtida introduzindo-se no subdomínio imaginário um alinhamento semiinfinito de dipolos, a partir do ponto que representa a posição refletida (imagem) do elemento real, extendendo-se para a direção contrária à fronteira (espelho). Baseado na expressão obtida por Keller [7], a relação entre os parâmetros dos elementos reais e dos elementos imagem é dada por:

$$
\sum \frac{\sigma^{\text {real }}}{i}+\sum\left[\frac{i}{\lambda} \sigma^{\text {imagem }} \int_{\bar{z}}^{\bar{z}+i \infty} e^{\frac{i}{\lambda}(\delta-\bar{z})} G^{*} d \delta\right],
$$

onde $\lambda$ é o coeficiente presente na condição do terceiro tipo, $\bar{z}$ é a posição da imagem e $G^{*}$ é a função que expressa a imagem do elemento.

$\mathrm{O}$ acoplamento do MI ao AEM permite a representação de contornos regionais com linhas, observando-se a validade da geometria segundo Keller [7]. Os contornos regionais usualmente constituem-se nos contornos externos do domínio de problemas de hidrogeologia e sua geometria pode ser representada por planos como descrito por Keller sem perda de detalhes na região dos contornos internos. O problema caracterizado no aqüífero Barreiras, abordado neste trabalho, apresenta contornos onde são conhecidas condições do primeiro e do segundo tipo.

\section{Implementação}

No presente estudo, utiliza-se a biblioteca AEM TimSL 0.3 (disponível na página http://www.engr.uga.edu/ mbakker). A implementação do Método de Imagens é 
desenvolvida de forma a impor a presença dos contornos regionais aos elementos implementados na biblioteca AEM. Dois módulos são para tanto assim especificados: o primeiro para a especificação e verificação dos espelhos segundo os critérios de aplicabilidade definidos por Keller [7]; o segundo para a construção dinâmica das imagens dos elementos de acordo com o elemento real e a condição de contorno especificada. A implementação do modelo de recarga, por sua vez, é feita definindo-se um módulo adicional internamente ao TimSL onde são especificadas as características da faixa de domínio, as propriedades do meio e a taxa de recarga regional.

\section{Aplicação}

O aqüífero Barreiras estende-se por uma longa faixa com largura de aproximadamente $30 \mathrm{~km}$ ao longo de grande parte da costa brasileira. A área de aplicação do modelo encontra-se na faixa litoral leste do Estado do Rio Grande do Norte onde encontra-se instalado um importante projeto de captação de água (Figura 3). Observa-se o fato de que a geometria da costa e do afloramento segue uma tendência retilínea durante um trecho bastante longo. Essas fronteiras podem, portanto ser aproximadas por linhas retas paralelas que definem a faixa onde é aplicado o modelo global definido pela equação (3.2). Na Figura 3, a região em branco define a oeste a região rochosa e a leste a costa. As fronteiras a oeste do aqǘfero definem a parede impermeável e a leste a linha drenante.

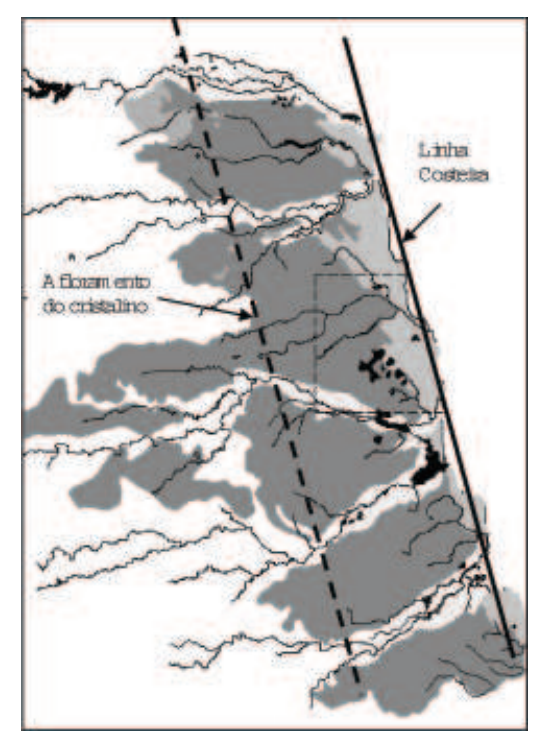

Figura 3: Aproximação dos contornos regionais do aqüífero Barreiras. A linha tracejada aproxima a parede impermeável, a linha cheia, indica fronteira drenante.

Observa-se na região duas formações predominantes: a Formação Barreiras (a oeste) e a Formação Dunas (próxima ao litoral). A Formação Dunas e a rede 
hidrográfica utilizadas representadas no modelo são representadas por Elementos Analíticos enquanto a recarga da Formação Barreiras e seus parâmetros hidráulicos são representada pela equação (3.2). Para o acoplamento de ambos modelos (Elementos Analíticos e recarga unidimensional), são aplicados elementos na região externa à faixa de domínio utilizando-se o Método de Imagens. Na Figura 4 é ilustrado o posicionamento desses elementos no modelo.

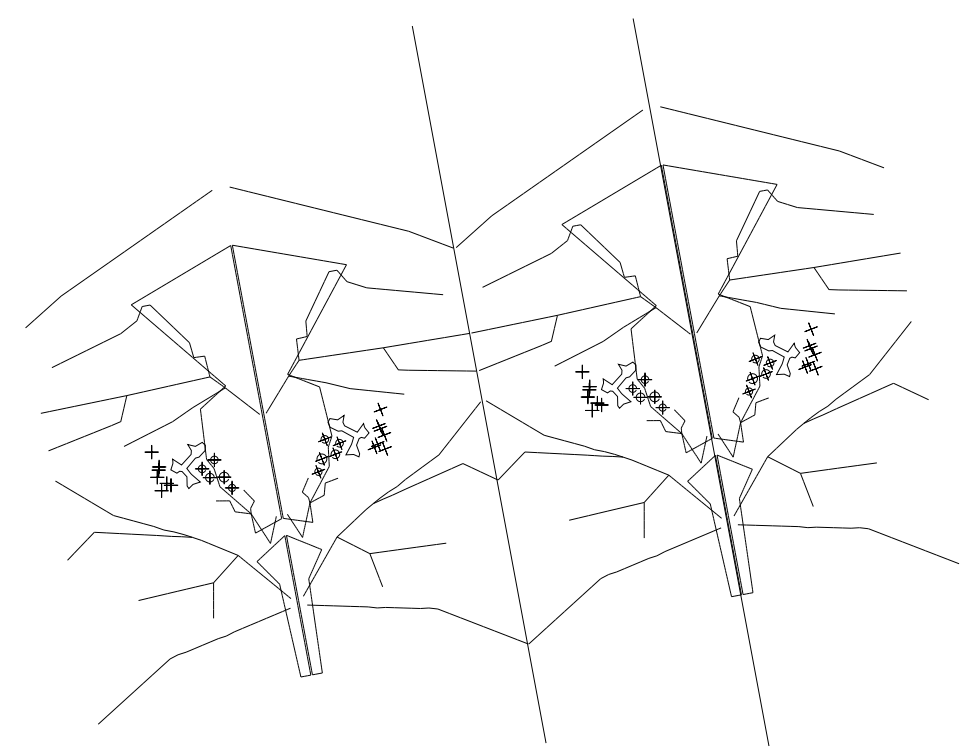

Figura 4: Posicionamento de elementos para o modelo. Linhas escuras (interior da faixa) correspondem ao domínio real da aplicação. As demais linhas representam as imagens utilizadas na solução

A distribuição de potenciais hidráulicos originalmente observada na região é apresentada na Figura 5 com linhas tracejadas. A distribuição resultante da instalação da captação no local é representada com linhas cheias. Os impactos na distribuição de potenciais são observados principalmente em torno da região a leste da lagoa, onde observa-se o rebaixamento de aproximadamente três metros.

\section{Conclusão}

Neste trabalho, o Método de Elementos Analíticos (AEM) foi empregado com o auxílio do Método de Imagens em um problema tipicamente encontrado em aqǘferos. O uso do Método de Imagens permite ao AEM ser utilizado com facilidade em domínios semi-infinitos, por exemplo na forma de faixa retilínea. Nesse caso, a recarga regional do aqǘfero Barreiras foi aproximada utilizando-se uma expressão unidimensional bastante simples. 


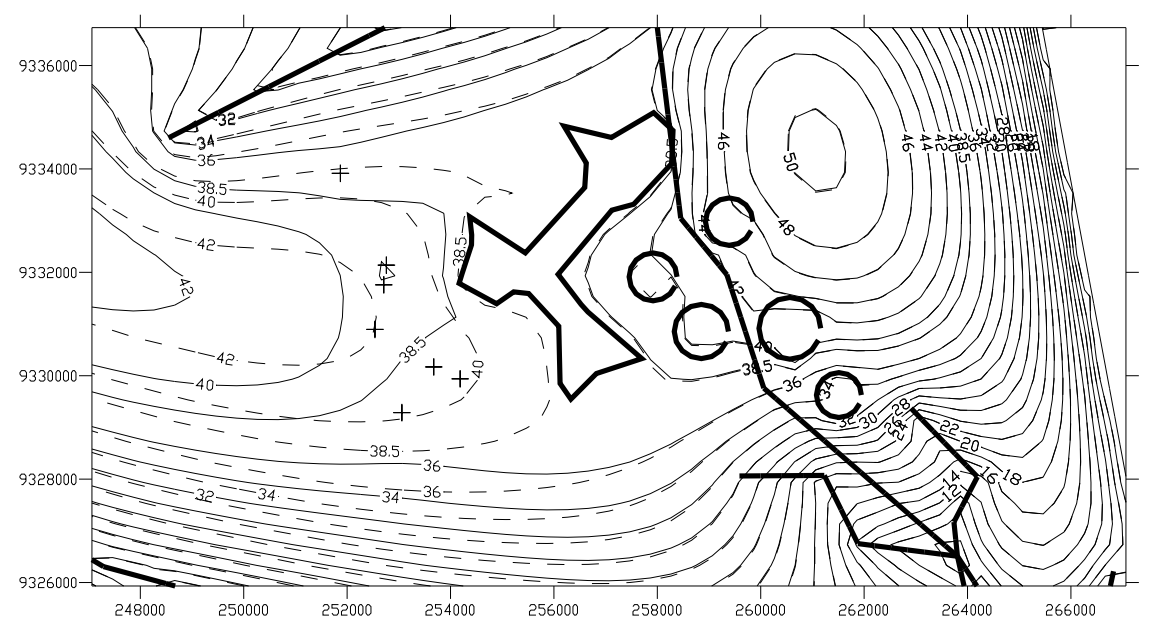

Figura 5: Distribuição de cargas hidráulicas na região da Lagoa do Bonfim. A linha tracejada indica condições naturais e a linha contínua, situação com bombeamento.

\section{Agradecimentos}

Os autores agradecem ao CNPq e à FAPESP (processo 02/09696-3) pelo suporte financeiro.

\section{Referências}

[1] E.I. Anderson, The method of images for leaky boundaries. Adv. Water Resour., 23 (2000),461-74.

[2] G. De Josselin de Jong, Singularity distribution for the analysis of multiple fluid flow through porous media, J. Geophys. Res., 65 (1960) 67-74.

[3] W.J. De Lange, NAGROM, a model for national groundwater management and regional and local studies, Eur. Water Pollut. Control, 6 (1996), 63-67.

[4] L. Fox e I.B. Parker, "Chebyshev Polynomials in Numerical Approximation", Oxford University Press, New York, 1968.

[5] H.M. Haitjema, "Analytic Element Modeling in Groundwater Flow", Academic, San Diego, 1995.

[6] I. Janković, "High-order analytic elements in modeling groundwater flow", Tese de Doutorado, Univ. of Minn., Minneapolis, 1997.

[7] J.B. Keller, The scope of the Image Method, Comm. on Pure and Appl. Math, 4 (1953), 505-512.

[8] M. Muskat, "The flow of homogeneous flow through porous media". McGrawHill, Ann Arbor., 1937 
[9] N.I. Muskhelishvili, "Some Basic Problems of the Mathematical Theory of Elasticity", Noordhoff, Groningen, 1953a.

[10] N.I. Muskhelishvili, "Singular Integral Equations", Noordhoff, Groningen, 1953b.

[11] P.Y. Polubarinova-Kochina, "Theory of the groundwater movement", Princeton Univ. Press, Princeton, 1962.

[12] O.D.L. Strack e H.M. Haitjema, Modeling double aquifer flow using a comprehensive potential and distributed singularities 1 . Solution for homogeneous permeability, Water Resour. Res., 17 (1981), 1535-1549;

[13] O.D.L. Strack e H.M. Haitjema, Modeling double aquifer flow using a comprehensive potential and distributed singularities 2. Solution for inhomogeneous permeabilities, Water Resour. Res, 17 (1981), 1551-1560;

[14] O.D.L. Strack, The theory and applications of the analytic element method, Reviews of Geophysics, 41 (2003), 1005-1020;

[15] O.D.L. Strack. "Groundwater mechanics", Prentice-Hall, Englewood Cliffs, 1989. 
